\title{
Application of $\frac{G^{\prime}}{G}-$ expansion method to two concert problems
}

\author{
Zainab Ayati*, Jafar Biazar \\ Department of Applied Mathematics, Faculty of Mathematical Science, University of Guilan, \\ P.O. Box 41635-19141, P.C. 41938336997, Rasht, Iran \\ *Corresponding author E-mail: Zainab.ayati@guilan.ac.ir
}

\begin{abstract}

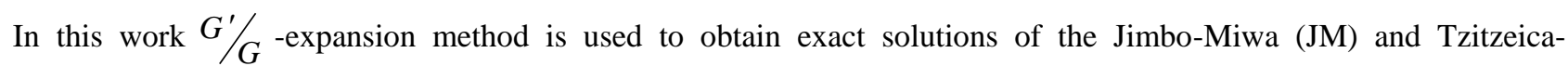

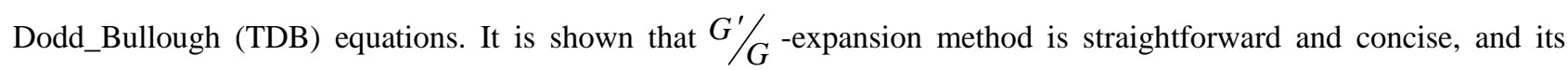
applications are promising.
\end{abstract}

Keywords: $G^{\prime} / G$-expansion method, Jimbo-Miwa equation, Tzitzeica-Dodd_Bullough equation, Exact solutions.

\section{Introduction}

The nonlinear equations of mathematical physics are major subjects in physics and engineering, and various powerful methods have been presented, such as the tanh method [1-2], sine-cosine method [3], homotopy perturbation method [4-5], variational iteration method [6-7], Adomian decomposition method [8], Exp-function method [9-11], and many others [12-13]. Very recently, Wang et al. [14] introduced a new method called the $G^{\prime} / G$ expansion method to look for travelling wave solutions of nonlinear evolution equations. The $G^{\prime} / G$ expansion method is based on the assumptions that the travelling wave solutions can be expressed by a polynomial in $G^{\prime} / G$ and that $G=G(\xi)$ satisfies a second order linear ordinary differential equation (ODE).

The present paper is motivated by the desire to extend the $G^{\prime} / G$ expansion method to the Jimbo-Miwa and TDB equations. Jimbo- Miwa equation is of particular interest in science and has been known in the following form

$$
u_{x x y}+3 u_{y} u_{x x}+3 u_{x} u_{x y}+2 u_{y t}-3 u_{x z}=0
$$

where $u=u(x, y, z, t)$. Eq. (1) is investigated by Jimbo and Miwa and its certain soliton solutions are obtained [15]. Then, it is studied by several authors regarding its solutions, symmetries and integrability properties [16-18]. Wazwaz $[19,20]$ employed the tanh-coth and Hirota's bilinear methods to solve Eq. (1).

The second equation that will be considered is the Tzitzeica-Dodd-Bullough (TDB) equation [21], in the following form

$$
u_{x t}=e^{-u}+e^{-2 u} \text {. }
$$

This equation plays a significant role in many scientific applications such as solid state physics, nonlinear optics and quantum field theory. TDB equation has been solved by using exp-function method in [22]. 


\section{The $\frac{G^{\prime}}{G}-$ expansion method}

Consider a nonlinear partial differential equation, in two independent variables say $x$ and $t$, in the form

$$
p\left(u, u_{t}, u_{x}, u_{x x}, u_{t t}, \ldots\right)=0 .
$$

Where $u=u(x, t)$ is an unknown function, $p$ is non-linear equation in $u=u(x, t)$ and its various partial derivatives. To apply the method following steps showed be fallowed.

Step 1. Using the transformation

$$
\xi=x-w t
$$

where $w$ is constant, we can rewrite Eq. (3) as the following nonlinear ODE:

$$
Q\left(u, u^{\prime}, u^{\prime \prime}, u^{\prime \prime \prime}, \ldots\right)=0 \text {. }
$$

Where the superscripts denote the derivatives with respect to $\xi$.

Step 2. Suppose that the solution of ODE (5) can be expressed by a polynomial in $G^{\prime} / G$ as follows:

$$
u(\xi)=\sum_{i=0}^{m} \alpha_{i}\left(\frac{G^{\prime}}{G}\right)^{i}
$$

where $G=G(\xi)$ satisfies the second order LODE in the form as fallows

$$
G^{\prime \prime}+\lambda G^{\prime}+\mu G=0
$$

$\alpha_{i}, \lambda$, and $\mu$ are constants to be determined later with $\alpha_{m} \neq 0$. The positive integer $m$ can be determined by considering the homogeneous balance the highest order derivatives and highest order nonlinear appearing in ODE (5).

Step 3. Substituting Eq. (6) into Eq. (5) and using the second order LODE, Eq. (7) yields an algebraic equation involving powers of $G^{\prime} / G$. Equating the coefficient of each power of $G^{\prime} / G$ to zero gives a set of algebraic equations for determining $\alpha_{i}, w, \lambda$, and $\mu$.

Step 4. Assuming that the constants $\alpha_{i}, w, \lambda$, and $\mu$ can be obtained by solving the algebraic equations in Step 3 . Since the general solutions of the second order LODE (7), depending on the sign of $\Delta=\lambda^{2}-4 \mu$, are well known for us, by substituting $\alpha_{i}, w$, and the general solutions of Eq. (7) into Eq. (6), solutions of the nonlinear evolution Eq. (3) can be obtained.

\section{Application to the JM equation}

To apply $G^{\prime} / G^{-e x p a n s i o n ~ m e t h o d ~ o n ~ E q . ~(1), ~ l e t ' s ~ i n t r o d u c e ~ a ~ v a r i a b l e ~} \xi$, defined as

$$
\xi=x+y+z-w t
$$

So, Eq. (1) turns to the following ordinary differential equation, 


$$
u^{(4)}+6 u^{\prime} u^{\prime \prime}-(2 w+3) u^{\prime \prime}=0
$$

where $w$ is constant to be determined. By integrating from Eq. (9), we obtain

$$
u^{\prime \prime \prime}+3 u^{\prime 2}-(2 w+3) u^{\prime}=c,
$$

where $c$ is an integration constant that is to be determined later.

Suppose that the solution of ODE Eq. (10) can be expressed by a polynomial in $G^{\prime} / G$ as follows:

$$
u(\xi)=\sum_{i=0}^{m} \alpha_{i}\left(\frac{G^{\prime}}{G}\right)^{i}
$$

where $G=G(\xi)$ satisfies the second order LODE (7). Balancing the terms $u^{\prime \prime \prime}$ and $u^{\prime 2}$ in Eq. (10), yields to $m=1$. So we can write (11) as the following simple form

$$
u(\xi)=\alpha_{1}\left(\frac{G^{\prime}}{G}\right)+\alpha_{0}, \quad \alpha_{1} \neq 0,
$$

By substituting (12) into Eq. (10) and collecting all terms with the same power of $G^{\prime} / G$ together, and equating each coefficient of the terms to zero, we derive a set of algebraic equations for determining $\alpha_{1}, \alpha_{0}, c, w, \lambda$, and $\mu$ as follows

$$
\begin{aligned}
& \left(\frac{G^{\prime}}{G}\right)^{0}: 3 \alpha_{1} \mu-\alpha_{1} \lambda^{2} \mu+3 \alpha_{1}^{2} \mu^{2}+2 \mathrm{w} \alpha_{1} \mu-\mathrm{c}=0, \\
& \left(\frac{G^{\prime}}{G}\right)^{1}:-8 \alpha_{1} \lambda \mu-\alpha_{1} \lambda^{3}+6 \alpha_{1}^{2} \lambda \mu+2 \mathrm{w} \alpha_{1} \lambda+3 \alpha_{1} \lambda=0, \\
& \left(\frac{G^{\prime}}{G}\right)^{2}:-8 \alpha_{1} \mu-7 \alpha_{1} \lambda^{2}+6 \alpha_{1}^{2} \mu+3 \alpha_{1}^{2} \lambda^{2}+2 \mathrm{w} \alpha_{1}+3 \alpha_{1}=0, \\
& \left(\frac{G^{\prime}}{G}\right)^{3}:-12 \alpha_{1} \lambda+6 \alpha_{1}^{2} \lambda=0, \\
& \left(\frac{G^{\prime}}{G}\right)^{4}:-6 \alpha_{1}+3 \alpha_{1}^{2}=0 .
\end{aligned}
$$

By the solution of these algebraic equations, the following results are obtained

$$
\alpha_{1}=2, w=-2 \mu+\frac{1}{2} \lambda^{2}-\frac{3}{2}, c=4 \mu \text {. }
$$

Where $\lambda, \mu$, and $\alpha_{0}$ are arbitrary constants.

By substituting (14) into (12), we drive

$$
u(\xi)=2\left(\frac{G^{\prime}}{G}\right)+\alpha_{0}
$$

where

$$
\xi=x+y+z-\left(-2 \mu+\frac{1}{2} \lambda^{2}-\frac{3}{2}\right) t .
$$

By substituting the general solutions of Eq. (7) into (15) we would have three types of traveling wave solutions of the JM equation as follows:

When $\lambda^{2}-4 \mu>0$, 


$$
u_{1}(\xi)=\sqrt{\lambda^{2}-4 \mu}\left(\frac{A \sinh \frac{1}{2} \sqrt{\lambda^{2}-4 \mu} \xi+B \cosh \frac{1}{2} \sqrt{\lambda^{2}-4 \mu} \xi}{A \cosh \frac{1}{2} \sqrt{\lambda^{2}-4 \mu} \xi+B \sinh \frac{1}{2} \sqrt{\lambda^{2}-4 \mu} \xi}\right)-\lambda+\alpha_{0} .
$$

While $\lambda^{2}-4 \mu<0$,

$$
u_{2}(\xi)=\sqrt{4 \mu-\lambda^{2}}\left(\frac{-A \sin \frac{1}{2} \sqrt{4 \mu-\lambda^{2}} \xi+B \cos \frac{1}{2} \sqrt{4 \mu-\lambda^{2}} \xi}{A \cos \frac{1}{2} \sqrt{4 \mu-\lambda^{2}} \xi+B \sin \frac{1}{2} \sqrt{4 \mu-\lambda^{2}} \xi}\right)-\lambda+\alpha_{0} .
$$

For $\lambda^{2}-4 \mu=0$,

$$
u_{3}(\xi)=\frac{2 B}{A+B \xi}-\lambda+\alpha_{0} .
$$

Where $A$ and $B$ are arbitrary constants and

$$
\xi=x+y+z-\left(-2 \mu+\frac{1}{2} \lambda^{2}-\frac{3}{2}\right) t .
$$

In particular case of $A \neq 0$, and $B=0$, Eqs. (17) and (18) turns to

$$
\begin{aligned}
& u(\xi)=\sqrt{\lambda^{2}-4 \mu} \tanh \left(\frac{1}{2} \sqrt{\lambda^{2}-4 \mu} \xi\right)-\lambda+\alpha_{0} . \\
& u(\xi)=-\sqrt{4 \mu-\lambda^{2}} \tan \left(\frac{1}{2} \sqrt{4 \mu-\lambda^{2}} \xi\right)-\lambda+\alpha_{0} .
\end{aligned}
$$

And for $A=0$, and $B \neq 0$, Eqs. (17) and (18) turns to

$$
\begin{aligned}
& u(\xi)=\sqrt{\lambda^{2}-4 \mu} \operatorname{coth}\left(\frac{1}{2} \sqrt{\lambda^{2}-4 \mu} \xi\right)-\lambda+\alpha_{0} . \\
& u(\xi)=\sqrt{4 \mu-\lambda^{2}} \cot \left(\frac{1}{2} \sqrt{4 \mu-\lambda^{2}} \xi\right)-\lambda+\alpha_{0} .
\end{aligned}
$$

\section{Application to the TDB equation}

By using the transformations

$$
v(x, t)=e^{-u(x, t)}, \xi=x-w t,
$$

Eq. (2) turns to an ordinary differential equation, as the following

$$
w\left(v v^{\prime \prime}-v^{\prime 2}\right)-v^{3}-v^{4}=0,
$$

and

$$
u=\arcsin h\left[\frac{v^{-1}-v}{2}\right] .
$$

Suppose that the solution of ODE Eq. (21) can be expressed by a polynomial in $G^{\prime} / G$ as shown in (11), where $G=G(\xi)$ satisfies the second order LODE (7). By considering the homogeneous balance between $v v^{\prime \prime}$ and $v^{4}$ in Eq. (21), we required that

$$
2 m+2=4 m,
$$

and 


$$
m=1 \text {. }
$$

So we can write (11) as the following simple form

$$
v(\xi)=\alpha_{1}\left(\frac{G^{\prime}}{G}\right)+\alpha_{0}, \quad \alpha_{1} \neq 0 .
$$

By substituting (24) into Eq. (21) and collecting all terms with the same power of $G^{\prime} / G$ together, the left-hand side of Eq. (21) is converted into another polynomial in $G^{\prime} / G$. Equating each coefficient of this polynomial to zero yields to a set of simultaneous algebraic equations for determining $\alpha_{1}, \alpha_{0}, w, \lambda$, and $\mu$ as follows:

$$
\begin{aligned}
& \left(\frac{G^{\prime}}{G}\right)^{0}:-\alpha_{0}^{3}+\mathrm{w} \alpha_{0} \alpha_{1} \lambda \mu-\mathrm{w} \alpha_{1}^{2} \mu^{2}-\alpha_{0}^{4}=0 \\
& \left(\frac{G^{\prime}}{G}\right)^{1}: 2 \mathrm{w} \alpha_{0} \alpha_{1} \mu+\mathrm{w} \alpha_{0} \alpha_{1} \lambda^{2}-\mathrm{w} \alpha_{1}^{2} \lambda \mu-3 \alpha_{0}^{2} \alpha_{1}-4 \alpha_{0}^{3} \alpha_{1}=0 \\
& \left(\frac{G^{\prime}}{G}\right)^{2}: 3 \mathrm{w} \alpha_{0} \alpha_{1} \lambda-3 \alpha_{0} \alpha_{1}^{2}-6 \alpha_{0}^{2} \alpha_{1}^{2}=0 \\
& \left(\frac{G^{\prime}}{G}\right)^{3}: 2 \mathrm{w} \alpha_{0} \alpha_{1}+\mathrm{w} \alpha_{1}^{2} \lambda-\alpha_{1}^{3}-4 \alpha_{0} \alpha_{1}^{3}=0 \\
& \left(\frac{G^{\prime}}{G}\right)^{4}: \mathrm{w} \alpha_{1}^{2}-\alpha_{1}^{4}=0
\end{aligned}
$$

Solving this algebraic equations above, yields to

$$
\alpha_{0}= \pm \frac{1}{2} \frac{1}{\sqrt{\lambda^{2}-4 \mu}} \lambda-\frac{1}{2}, \alpha_{1}= \pm \frac{1}{\sqrt{\lambda^{2}-4 \mu}}, w=\frac{1}{\lambda^{2}-4 \mu} .
$$

Where $\lambda$ and $\mu$ are arbitrary constants.

By substituting (26) into (24), we drive

$$
v(\xi)= \pm \frac{1}{\sqrt{\lambda^{2}-4 \mu}}\left(\frac{G^{\prime}}{G}\right) \pm \frac{1}{2} \frac{1}{\sqrt{\lambda^{2}-4 \mu}} \lambda-\frac{1}{2},
$$

Where

$$
\xi=x-\frac{1}{\lambda^{2}-4 \mu} t
$$

By substituting the general solutions of Eq. (7) into (27), three types of traveling wave solutions of the TDB equation have been obtained:

When $\lambda^{2}-4 \mu>0$,

$$
v_{1,2}(\xi)= \pm \frac{1}{2}\left(\frac{A \sinh \frac{1}{2} \sqrt{\lambda^{2}-4 \mu} \xi+B \cosh \frac{1}{2} \sqrt{\lambda^{2}-4 \mu} \xi}{A \cosh \frac{1}{2} \sqrt{\lambda^{2}-4 \mu} \xi+B \sinh \frac{1}{2} \sqrt{\lambda^{2}-4 \mu} \xi}\right)-\frac{1}{2}
$$

While $\lambda^{2}-4 \mu<0$

$$
v_{3,4}(\xi)= \pm \frac{1}{2}\left(\frac{-A \sin \frac{1}{2} \sqrt{4 \mu-\lambda^{2}} \xi+B \cos \frac{1}{2} \sqrt{4 \mu-\lambda^{2}} \xi}{A \cos \frac{1}{2} \sqrt{4 \mu-\lambda^{2}} \xi+B \sin \frac{1}{2} \sqrt{4 \mu-\lambda^{2}} \xi}\right)-\frac{1}{2} .
$$

In particular case of $A \neq 0, B=0, \quad$ and $c=\frac{1}{-4 \mu}$, Eq. (29) turns to 


$$
v(x, t)=-\frac{1}{2}\left(1 \pm \tanh \left(\frac{1}{2 \sqrt{c}}(x-c t)\right)\right) .
$$

This is the same as the result obtained by wazwaz [21].

\section{Conclusion}

In this article, $G^{\prime} / G$-expansion method is used to obtain exact solutions of the Jimbo-Miwa and TDB equations. The solutions being determined in this paper are more general, and it is not difficult to arrive at some known analytic

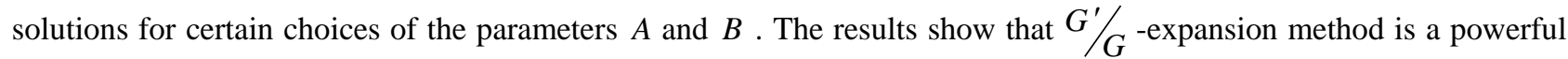
tool for obtaining exact solution. Applications of $G^{\prime} / G$-expansion method for some equations are under study in our research group. The computations associated in this work were performed by using Maple 13.

\section{References}

[1] A.M. Wazwaz, The tanh method: Exact solutions of the Sine-Gordon and Sinh- Gordon equations, Applied Mathematics and Computation 167 (2005)1196-1210.

[2] Malfliet W, Hereman W. The tanh method: I. Exact solutions of nonlinear evolution and wave equations, Physica Scripta 54 (1996) 563-8.

[3] Wazwaz AM. The tanh and the sine-cosine methods for a reliable treatment of the modified equal width equation and its variants, Communications in Nonlinear Science and Numerical Simulation 11 (2006) 148-60.

[4] J. Biazar, H. Ghazvini, Exact solutions for non-linear Schrödinger equations by He's homotopy perturbation method, Physics Letters A 366 (2007) 79-84.

[5] J.H. He, Application of homotopy perturbation method to nonlinear wave equations, Chaos Solitons Fractals 26(2005) 695-700.

[6] J.H. He, Variational iteration method - a kind of non-linear analytical technique: some examples, International Journal of Non-Linear Mechanics 34 (1999) 699-708.

[7] J.H. He, Variational iteration method for autonomous ordinary differential systems, Applied Mathematics and Computation 114 (2000) 115123.

[8] J. Biazar, E. Babolian, A. Nouri, R. Islam, An alternate algorithm for computing Adomian Decomposition method in special cases, Applied Mathematics and Computation 38 (2-3) (2003) 523- 529.

[9] J.H. He, X.H. Wu, Exp-function method for nonlinear wave equations, Chaos Solitons Fractals 30 (2006) 700-708.

[10] S. Zhang, Application of Exp-function method to a KdV equation with variable coefficients, Physics Letters A 365 (2007) 448-453.

[11] J. Biazar, z. ayati, Application of Exp-function method to Equal-width wave equation, Physica Scripta (2008) 78045005.

[12] M.L. Wang, Exact solutions for a compound KdV-Burgers equation, Physics Letters A 213 (1996) 279.

[13] M.A. Abdou, The extended F-expansion method and its application for a class of nonlinear evolution equations, Chaos Solitons Fractals 31 (2007) 95-104.

[14] M.L. Wang, X.Z. Li, J.L. Zhang, The $G^{\prime} / G$ expansion method and travelling wave solutions of nonlinear evolution equations in mathematical physics, Physics Letters A 372 (2008) 417-423.

[15] M. Jimbo, T. Miwa, Solitons and infinite-dimensional Lie algebras, Publ. Res. Inst. Math. Sci. 19 (1983) 943.

[16] W.X. Ma, Comment on "Generalized $\mathrm{W}_{\infty}$ symmetry algebra of the conditionally integrable nonlinear evolution equation, Journal of Mathematical Physics 40 (1999) 3685.

[17] B. Dorizzi, B. Grammaticos, A. Ramani, P. Winternitz, Are all the equations of the Kadomtsev-Petviashvili hierarchy integrable?, Journal of Mathematical Physics 27 (1986) 2848.

[18] S.Y. Lou, J.P. Weng, Generalized $\mathrm{W}_{\infty}$ symmetry algebra of the conditionally integrable nonlinear evolution equation, Journal of Mathematical Physics 36 (1995) 3492

[19] A.M. Wazwaz, New solutions of distinct physical structures to high-dimensional nonlinear evolution equations, Applied Mathematics and Computation 196 (2008) 363

[20] A.M. Wazwaz, Multiple-soliton solutions for the Calogero-Bogoyavlenskii-Schiff, Jimbo-Miwa and YTSF equations, Applied Mathematics and Computation 203 (2) (2008) 592

[21] A.M.Wazwaz, The tanh method: solitons and periodic solutions for the Dodd-Bullough-Mikhailov and the Tzitzeica-Dodd-Bullough equations, Chaos, Solitons \& Fractals 2005;25(1):55-65

[22] J.H. He, M.A. Abdou New periodic solutions for nonlinear evolution equations using Exp-function method, Chaos, Solitons and Fractals 34 (2007) 1421-1429. 